\title{
CARACTERIZAÇÃO SOCIODEMOGRÁFICA- CLİNICA E DIAGNÓSTICOS DE ENFERMAGEM NA ENFERMARIA PEDIÁTRICA
}

Suelen Reiniack ${ }^{1}$, Jamile Pascoal Franco Gonçalves ${ }^{1}$, Alexandre Sousa da Silva ${ }^{1}$, Teresa Tonini ${ }^{1}$

Objetivo: caracterizar o perfil de pacientes internados na enfermaria de pediatria e identificar os Diagnósticos de Enfermagem mais prevalentes, de acordo com a taxonomia NANDA-I. Metodologia: estudo descritivo realizado a partir de prontuários de 100 pacientes. Foram consideradas variáveis como: idade, gênero, sistema de classificação de pacientes, diagnóstico clínico e de enfermagem. Utilizou-se o programa Rcommander® para realização da análise exploratória e testes de hipóteses nãoparamétricos, sendo considerado nível de significância de 5\%. Resultado: predominância do sexo masculino (71\%), idade média de 7,32 anos, o Cuidado Intermediário (49\%) foi o mais frequente, Pediatria foi a clínica com maior número de internações (39\%), encontrados 44 diagnósticos médicos e 46 diagnósticos de enfermagem, sendo 27 considerados preponderantes. Conclusão: a realização do diagnóstico situacional possibilita um caráter norteador para as demais etapas do Processo de Enfermagem e direciona as ações de cuidado, contribuindo para a melhora contínua da qualidade e segurança dos cuidados.

Descritores: Processo de Enfermagem, Diagnósticos de Enfermagem, Enfermagem Pediátrica.

\section{CLINICAL SOCIODEMOGRAPHICALAND DIAGNOSTIC CHARACTERIZATION OF NURSING IN PEDIATRIC NURSING}

Ojective: to characterize the profile of hospitalized patients in the pediatric ward and to identify the most prevalent Nursing Diagnoses, according to the NANDA-I taxonomy. Methodology: descriptive study based on medical records of 100 patients. Variables such as: age, gender, patient classification system, clinical and nursing diagnosis were considered. The Rcommander ${ }^{\circledR}$ program was used to perform the exploratory analysis and tests of non-parametric hypotheses, being considered level of significance of 5\%. Results: predominance of males (71\%), mean age of 7.32 years, Intermediate Care (49\%) was the most frequent, Pediatrics was the clinic with the greatest number of hospitalizations (39\%), 44 medical and 46 nursing diagnoses, of which 27 were considered as preponderant. Conclusion: the accomplishment of the situational diagnosis allows a guiding character for the other stages of the Nursing Process and directs care actions, contributing to the continuous improvement of quality and safety of care.

Descriptors: Nursing Process; Nursing Diagnostics; Pediatric Nursing.

\section{CARACTERIZACIÓN SOCIODEMOGRÁFICA Y DIAGNÓSTICA CLÍNICA DE ENFERMERÍA EN ENFERMERÍA PEDIÁTRICA}

Objetivo: caracterizar el perfil de pacientes internados en la enfermería de pediatría e identificar los Diagnósticos de Enfermería más prevalentes, de acuerdo con la taxonomía NANDA-I. Metodología: estudio descriptivo realizado a partir de prontuarios de 100 pacientes. Se consideraron variables como: edad, género, sistema de clasificación de pacientes, diagnóstico clínico y de enfermería. Se utilizó el programa Rcommander® para realizar el análisis exploratorio y pruebas de hipótesis no paramétricas, siendo considerado nivel de significancia del 5\%. Resultado: En la mayoría de los casos, el cuidado intermedio (49\%) fue el más frecuente, la Pediatría fue la clínica con mayor número de internaciones (39\%), encontrados 44 diagnósticos médicos y, 46 diagnósticos de enfermería, siendo 27 considerados preponderantes. Conclusión: la realización del diagnóstico situacional posibilita un carácter orientador para las demás etapas del proceso de enfermería y dirige las acciones de cuidado, contribuyendo a la mejora continua de la calidad y seguridad del cuidado.

Descriptores: Proceso de Enfermería; Diagnósticos de Enfermería; Enfermería Pediátrica. 


\section{INTRODUÇÃO}

Maior atenção dos enfermeiros brasileiros começou a ser direcionada para a Sistematização da Assistência de Enfermagem (SAE) com os trabalhos de Wanda Horta, nos anos 60, com ênfase no planejamento da assistência, na tentativa de tornar autônoma a profissão e alcançar o estado de ciência. Sabe-se que a implantação da SAE é indispensável e desafiante para a realidade específica das instituições, favorecendo a consolidação da estrutura e processos organizacionais, em especial dos serviços e dos cuidados de enfermagem baseados em evidências clínicas e gerenciais científicas ${ }^{(1,2,3)}$.

A Resolução COFEN no 358/2009 reforça a necessidade de implantação da SAE, no sentido em que requer a análise do ambiente organizacional. Esse diagnóstico situacional consiste em uma das etapas de gestão disponiveis para pesquisa das condições e posterior planejamento das ações devendo ser utilizado para conhecimento do perfil da clientela assistida com objetivo de oferecer assistência mais eficaz e eficiente em prol da qualidade ${ }^{(4,5,2)}$.

A SAE norteia o trabalho profissional quanto aos eixos Método, Pessoas e Instrumentos, tornando possivel a eficiente implementação do Processo de Enfermagem (PE). Fundamentado em método científico, há adoção de estratégia de trabalho voltada à identificação de situações de saúde-doença, com oferecimento de ações de assistência de Enfermagem contributivas para a promoção, recuperação e reabilitação da saúde, além de prevenção de agravos do indivíduo, família e comunidade ${ }^{(4)}$.

O PE é um dos componentes específicos do eixo Método da SAE, como instrumento metodológico para orientação do cuidado profissional e documentação da prática, possibilitando a abordagem holística do paciente e o aprimoramento contínuo na oferta de cuidados de enfermagem. Apresenta-se organizado em cinco etapas inter-relacionadas, interdependentes e recorrentes compreendidas por Histórico de Enfermagem, Diagnóstico de Enfermagem (DE), Planejamento de Enfermagem, Implementação e Avaliação de Enfermagem(4).

Deve-se estar alerta para fatores influenciadores que dificultam a implantação do PE, tais como: ausência de caracterização do perfil do setor; inexistência de padronização da linguagem; falta de domínio para o uso do $\mathrm{PE}$; visão dos profissionais sobre o PE apenas como mais uma tarefa a ser realizada; subdimensionamento de pessoal; sobrecarga de trabalho da equipe de enfermagem; precárias condições estruturais dos serviços; baixa remuneração dos profissionais; além de fatores politicos e sociais(6.7.8).

O Processo de Enfermagem baseado em sistemas de linguagem padronizada requer a determinação do perfil da clientela e o conhecimento dos diagnósticos de enfermagem mais prevalentes desse dado grupo. A taxonomia da North American Nursing Diagnoses Association (NANDA-I) representa uma linguagem bastante difundida e utilizada no Brasil, permitindo a constituição de DE em diversas instituições hospitalares. Os DE estabelecidos com alto nível de coerência em relação aos componentes da característica definidora, fator relacionado ou fator de risco, necessitam ser identificados para que o cuidado possa ser direcionado à solução desses problemas ou redução da gravidade ou risco de ocorrência ${ }^{(9,10,11)}$.

Apesar de evidências de alguns obstáculos institucionais e gerenciais, a implementação da SAE demonstra melhora efetiva da qualidade da assistência prestada ao paciente. Neste sentido, a utilização dos diagnósticos e prescrição de cuidados de enfermagem representam a mudança de cultura na instituição e o rompimento com o modelo biomédico(3).

\section{OBJETIVO}

Analisar o perfil sociodemográfico e clínico de pacientes internados na enfermaria de pediatria e determinar os DE mais prevalentes, de acordo com a taxonomia da NANDA-I.

\section{METODOLOGIA}

\section{Tipo de estudo}

Trata-se de um estudo descritivo, documental e seccional com abordagem quantitativa.

\section{Participantes da pesquisa}

A população do estudo foi constituída por 100 pacientes internados na enfermaria de pediatria, no periodo de dezembro de 2017 a julho de 2018.

\section{Local do estudo}

Realizado na enfermaria de pediatria de um hospital público no município do Rio de Janeiro. Essa enfermaria possui capacidade para 24 leitos, dos quais sete são destinados a pacientes clínicos pediátricos, sete destinados a pacientes com distúrbios onco-hematológicos e 10 leitos destinados a pacientes cirúrgicos eletivos no pré e pósoperatório de cirurgias de pequeno e médio porte, com faixa etária de um dia de vida aos 18 anos de idade.

\section{Coleta dos dados}

A partir de um de banco de dados relacionados à gestão da referida enfermaria de pediatria, buscou-se as variáveis idade, gênero, sistema de classificação de pacientes, diagnóstico médico e de enfermagem. A coleta 
foi realizada nos meses de agosto a outubro de 2018, por meio de formulário estruturado elaborado pelos próprios pesquisadores deste estudo.

\section{Procedimentos de análise dos dados}

Utilizou-se o software Excel ${ }^{\circledR}$ para tabulação dos dados e o programa Rcommander ${ }^{\circledR}$ versão 3.4.4 para elaboração da análise exploratória e testes de hipóteses. Decidiuse por testes não paramétricos (Teste Exato de Fisher, Wilcoxon e Kruskal-Wallis), após a realização de teste de normalidade de Shapiro -Wilk. Para identificação dos DE mais prevalentes foram considerados 27 preponderantes, que apresentassem o valor maior que $10 \%$ de frequência.

\section{Procedimentos éticos}

Apesquisa foi aprovada pelo Comitê de Ética em Pesquisa da Universidade Federal do Estado do Rio de Janeiro, em 01 de agosto de 2018, sob o Parecer no 2.793.648.

\section{RESULTADOS}

A tabela 1 apresenta a análise das características das variáveis idade, sexo, Sistema de Classificação de Pacientes (SCP) e clínica de internação:

Tabela 1 - Perfil dos pacientes internados na enfermaria de pediatria. Rio de Janeiro, (RJ), Brasil, 2018.

\begin{tabular}{|c|c|c|}
\hline Variáveis & $\mathbf{N}$ & $\%$ \\
\hline \multicolumn{3}{|l|}{ Sexo } \\
\hline Feminino & 29 & $29 \%$ \\
\hline Masculino & 71 & $71 \%$ \\
\hline \multicolumn{3}{|l|}{ Idade } \\
\hline Média & 7,32 & \\
\hline Idade mínima & 0 & \\
\hline Idade máxima & 18 & \\
\hline
\end{tabular}

\begin{tabular}{|c|c|c|}
\hline \multicolumn{3}{|l|}{$\begin{array}{c}\text { Sistema de Classi- } \\
\text { ficação } \\
\text { de Pacientes }\end{array}$} \\
\hline Cuidados Mínimos & 35 & $35 \%$ \\
\hline $\begin{array}{c}\text { Cuidados Interme- } \\
\text { diários }\end{array}$ & 49 & $49 \%$ \\
\hline $\begin{array}{l}\text { Cuidados Alta de- } \\
\text { pendência }\end{array}$ & 16 & $16 \%$ \\
\hline \multicolumn{3}{|l|}{$\begin{array}{c}\text { Clinica de Inter- } \\
\text { nação }\end{array}$} \\
\hline Cirurgia Pediátrica & 33 & $33 \%$ \\
\hline Onco-hematologia & 28 & $28 \%$ \\
\hline Pediatria & 39 & $39 \%$ \\
\hline
\end{tabular}

O gráfico 1 apresenta a distribuição da idade entres os gêneros masculino (Masc) e feminino (Fem). Os pacientes do sexo feminino apresentaram média de idade de 7,54 anos e desvio padrão de 5,25, já para o sexo masculino a idade média foi 7,27 e desvio padrão de 5,28. Por meio do teste de Wilcoxon e com p_valor 0,37 pode-se concluir que não existe diferença estatisticamente significativa das idades entre os gêneros:

Gráfico 1- Boxplot da distribuição das variáveis idade e gênero dos pacientes internados na enfermaria de pediatria. Rio de Janeiro, (RJ), Brasil, 2018.

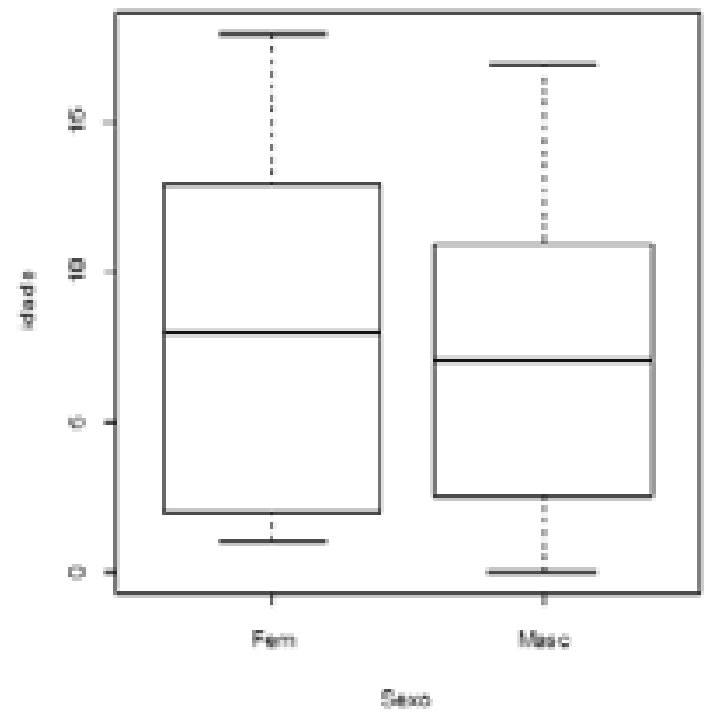

O gráfico 2 apresenta o boxplot da idade por classificação dos cuidados. Percebe-se que pacientes com Cuidados Mínimos (média 7,30, desvio 5,30) são em média mais velhos que os pacientes com Cuidados Intermediários (média 7,20, desvio 5,33) e de Alta de dependência (média 7,38, desvio 5,30). Por meio do teste de Kruskal-Wallis que obteve p_valor 0,0000005487, conclui-se que há ao menos um grupo com idade diferente dos demais:

Gráfico 2 - Boxplot da distribuição das variáveis idade e SCP dos pacientes internados na enfermaria de pediatria. Rio de Janeiro, (RJ), Brasil, 2018.

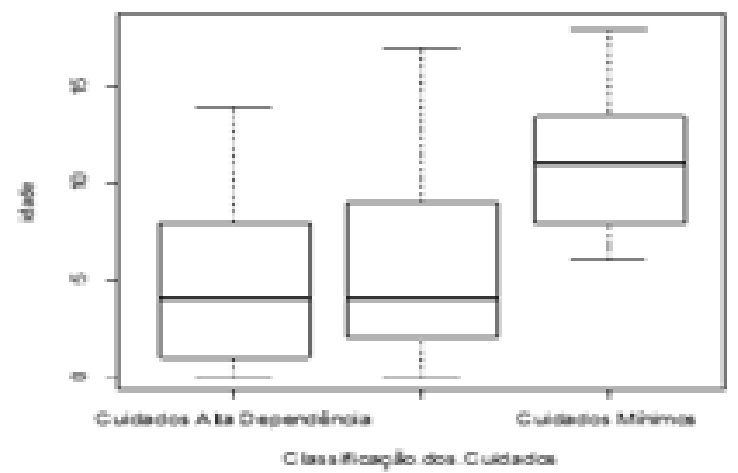

Enferm. Foco 2019: 127-134 
A Tabela 2 apresenta a frequência absoluta entre a clínica onde o paciente foi internado e SCP. Por meio do Teste Exato de Fisher e p valor 0,00009014 rejeita-se a hipóteses de independência entre a clínica de internação e o SCP.

Tabela 2 - Tabela de contingência de dupla entrada das variáveis Clínica de Internação e SCP. Rio de Janeiro, (RJ), Brasil, 2018.

$\begin{array}{cccc}\begin{array}{c}\text { Clinica de } \\ \text { Internação }\end{array} & \begin{array}{c}\text { Cuidados Alta } \\ \text { Dependência }\end{array} & \begin{array}{c}\text { Cuidados In- } \\ \text { termediários }\end{array} & \begin{array}{c}\text { Cuidados } \\ \text { Mínimos }\end{array} \\ \begin{array}{c}\text { Cirurgia pedi- } \\ \text { átrica }\end{array} & 2 & 20 & 12 \\ \begin{array}{c}\text { Onco-hema- } \\ \text { tologia }\end{array} & 1 & 10 & 17 \\ \text { Pediatria } & 13 & 19 & 6\end{array}$

Verificou-se o total de 44 diagnósticos médicos. Após agrupamento, determinou-se os prevalentes, sendo 13 (33\%) diagnósticos de doenças respiratórias, seguidos de 11 (28\%) de doenças crônicas, 10 (26\%) de encefalopatias, e cinco (13\%) de doenças infectocontagiosas na clínica de internação da Pediatria. Na clínica da CIPE prevaleceram as cirurgias de fimose, 11 (34\%), seguidas das gastrointestinais, sete (21\%), outras cirurgias diversas, sete (21\%), hérnias inguinais ou umbilicais, cinco (15\%), e colocação/retirada de cateter profundo, três (9\%). Na clínica de Onco-hematologia, prevaleceu a neutropenia febril, nove (32\%), seguida das internações para bloco de quimioterapia, oito (29\%), anemias, cinco (18\%), investigação hematológica, cinco (18\%), e outros, um $(3 \%)$.

A tabela 3 apresenta os 46 diagnósticos de enfermagem diferentes baseados na NANDA-I, sendo 18 relacionados aos diagnósticos de riscos e 28 relacionados aos diagnósticos com foco no problema. Identificou-se a média de oito DE por paciente:

Tabela 3 - Distribuição dos títulos diagnósticos de enfermagem identificados em pacientes internados na enfermaria de pediatria $(\mathrm{N}=100)$, de acordo com os domínios da taxonomia da NANDA-I. Rio de Janeiro, (RJ), Brasil, 2018

$\begin{array}{lll}\text { Dominio } & \begin{array}{l}\text { Diagnósticos de } \\ \text { enfermagem }\end{array} & \text { N (\%) } \\ \begin{array}{ll}\text { 1. Promoção da } \\ \text { Saúde }\end{array} & \text { Proteção ineficaz } & 39(39)\end{array}$

\begin{tabular}{|c|c|c|}
\hline \multirow{9}{*}{ 2. Nutrição } & $\begin{array}{l}\text { Volume de liquidos } \\
\text { excessivo }\end{array}$ & $26(26)$ \\
\hline & $\begin{array}{l}\text { Risco de glicemia } \\
\text { instável }\end{array}$ & $22(22)$ \\
\hline & $\begin{array}{l}\text { Nutrição desequi- } \\
\text { librada: menor do } \\
\text { que as necessidades } \\
\text { corporais }\end{array}$ & $21(21)$ \\
\hline & $\begin{array}{l}\text { Risco de desequili- } \\
\text { brio eletrolítico }\end{array}$ & $8(8)$ \\
\hline & $\begin{array}{l}\text { Padrão ineficaz de } \\
\text { alimentação do } \\
\text { lactente }\end{array}$ & $8(8)$ \\
\hline & $\begin{array}{l}\text { Volume de líquidos } \\
\text { deficiente }\end{array}$ & $5(5)$ \\
\hline & Sobrepeso & $l(1)$ \\
\hline & $\begin{array}{l}\text { Amamentação } \\
\text { interrompida }\end{array}$ & $1(1)$ \\
\hline & Icterícia neonatal & $1(1)$ \\
\hline \multirow{5}{*}{$\begin{array}{l}\text { 3. Eliminação e } \\
\text { Troca }\end{array}$} & $\begin{array}{l}\text { Troca de gases } \\
\text { prejudicada }\end{array}$ & $13(13)$ \\
\hline & Constipação & $12(12)$ \\
\hline & $\begin{array}{l}\text { Motilidade gastrin- } \\
\text { testinal disfuncional }\end{array}$ & $4(4)$ \\
\hline & $\begin{array}{l}\text { Eliminação urinária } \\
\text { prejudicada }\end{array}$ & $4(4)$ \\
\hline & Diarreia & $2(2)$ \\
\hline \multirow{9}{*}{$\begin{array}{l}\text { 4. Atividade/Re- } \\
\text { pouso }\end{array}$} & $\begin{array}{l}\text { Mobilidade fisica } \\
\text { prejudicada }\end{array}$ & $46(46)$ \\
\hline & Fadiga & $31(31)$ \\
\hline & $\begin{array}{l}\text { Risco de perfusão } \\
\text { tissular cerebral } \\
\text { ineficaz }\end{array}$ & $31(31)$ \\
\hline & $\begin{array}{l}\text { Mobilidade no leito } \\
\text { prejudicada }\end{array}$ & $17(17)$ \\
\hline & $\begin{array}{l}\text { Risco de perfusão } \\
\text { gastrintestinal } \\
\text { ineficaz }\end{array}$ & $16(16)$ \\
\hline & $\begin{array}{l}\text { Risco de sindrome } \\
\text { do desuso }\end{array}$ & $12(12)$ \\
\hline & $\begin{array}{l}\text { Risco de perfusão } \\
\text { renal ineficaz }\end{array}$ & $4(4)$ \\
\hline & $\begin{array}{l}\text { Risco de função } \\
\text { cardiovascular } \\
\text { prejudicada }\end{array}$ & $2(2)$ \\
\hline & Insônia & $1(1)$ \\
\hline
\end{tabular}




\begin{tabular}{lll} 
& $\begin{array}{l}\text { Risco de confusão } \\
\text { aguda }\end{array}$ & $29(29)$ \\
\hline $\begin{array}{l}\text { 5. Percepção/Cog- } \\
\text { nição }\end{array}$ & $\begin{array}{l}\text { Comunicação verbal } \\
\text { prejudicada }\end{array}$ & $16(16)$ \\
& $\begin{array}{l}\text { Conhecimento } \\
\text { deficiente }\end{array}$ & $10(10)$
\end{tabular}

$\begin{array}{ll}\text { 6. Autopercepção } & \begin{array}{l}\text { Distúrbio da imagem } \\ \text { corporal }\end{array}\end{array}$

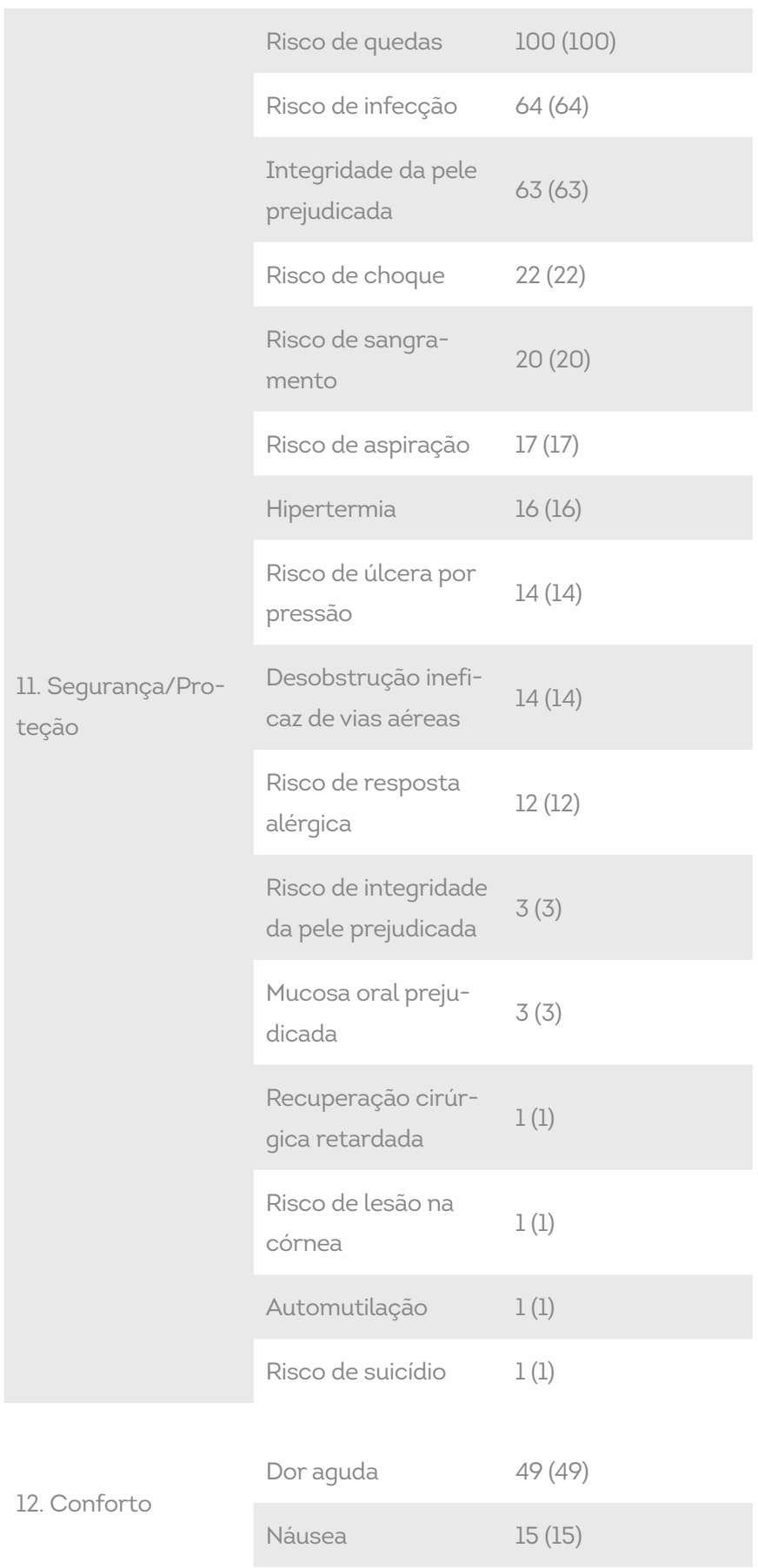

\section{DISCUSSÃO}

Quanto ao perfil sociodemográfico, identificouse porcentagem maior do sexo masculino 71 (71\%). Tal diferença entre os sexos era esperada, já que a cirurgia de fimose, que acomete indivíduos do sexo masculino, foi a que apresentou maior frequência entre as cirurgias pediátricas, com 11 (34\%) ocorrências. Outro fator a ser observado é a ocorrência de neoplasias, que, segundo estudos, são predominantes no sexo masculino, com exceção das leucemias. Neste estudo, 13 (46\%) dos pacientes portadores de neoplasias eram do sexo masculino, enquanto quatro ( $14 \%$ ) eram do sexo feminino ${ }^{(12)}$

A média de idade dos pacientes foi 7,32 anos (desvio padrão 5,27), com amplitude de 18 anos, compreendendo desde o primeiro dia de vida até os 18 anos. Por meio do teste de Wilcoxon, percebe que não há diferença estatisticamente significa da idade entre os gêneros.

Quanto ao SCP, a gestão da unidade utiliza a escala da DINI (2007). Neste estudo, o Cuidado Intermediário foi a variável mais frequente, com 49 (49\%) ocorrências, indo ao encontro das expectativas para uma unidade de internação, seguido do Cuidado Mínimo, 35 (35\%), e do Cuidado de Alta Dependência, 16 (16\%)

O SCP é uma ferramenta para tomada de decisão no processo gerencial em unidades de internação pediátrica que possibilita a classificação de pacientes neonatais e pediátricos em graus de dependência da enfermagem, subsidiando melhor alocação de recursos humanos, favorecendo a melhoria da qualidade assistencial e monitoramento de custos da assistência de enfermagem ${ }^{(13)}$.

Cabe ressaltar que, em uma unidade de internação em pediatria, todo recém-nascido e criança menor de seis anos deve ser classificado, no mínimo, na categoria de cuidado intermediário, independentemente da presença do acompanhante. No Cuidado Intermediário o paciente está estável sob o ponto de vista clínico e de enfermagem, acompanhado em período integral pela mãe ou responsável, em conjunto com a(o) qual realiza ações de autocuidado, mas requer auxílio da enfermagem para seu autocuidado e/ou apoio para o enfrentamento da situação de doença e hospitalização(14,8,13).

Por meio do teste de Kruskal-Wallis conclui-se que há diferença estatisticamente significativa no comportamento das idades entre as categorias de SCP. Gráfico 2 apresenta o boxplot da idade por SCP e permite observar que os pacientes que requerem cuidados intermediários e de alta dependência são em geral os mais novos. 
Observa-se na Tabela 2 a prevalência de cuidados intermediários, com 20 (61\%) ocorrências, seguidos de 11 (33\%) ocorrências de cuidados mínimos e dois (6\%) de cuidados de alta dependência na clínica de internação CIPE. Na clínica de internação de Onco-hematologia prevaleceram os cuidados mínimos, com 17 (61\%) ocorrências, seguidos dos cuidados intermediários, com $10(36 \%)$ e dos cuidados de alta dependência, com uma (3\%) ocorrência. Em relação à clínica de internação da Pediatria, prevaleceram os cuidados intermediários, 19 (49\%), seguidos dos cuidados de alta dependência, 13 (33\%), e cuidados mínimos, sete (18\%).

Destaca-se que na clínica de Onco-hematologia prevaleceram os pacientes que requeriam cuidados mínimos. Apesar de tratar pacientes com câncer, muitas vezes as ações de enfermagem no contexto da Onco-hematologia são mínimas, compreendendo medidas para minimizar a dor, dar suporte psicológico, promover um ambiente confortável, além de proceder diante dos efeitos colaterais dos quimioterápicos. A oncologia envolve questões de enfrentamento, como a finitude, as mutilações, a agressividade terapêutica e as perspectivas da utilização de terapêuticas antineoplásicas ${ }^{(12)}$.

Quanto aos diagnósticos médicos verificou-se a prevalência das cirurgias de fimose, 11 (34\%), seguidas das doenças respiratórias, $13(33 \%)$ e a neutropenia febril, nove (32\%).

É necessário o conhecimento acerca dos diagnósticos clínicos e de enfermagem prevalentes na enfermaria de pediatria de acordo com a realidade da instituição para agilizar o trabalho do enfermeiro, detectando precocemente possiveis agravos, além de diminuir os riscos de morbimortalidade. Para determinar os DE prevalentes utilizou-se os diagnósticos com frequência maior que 10\%, resultando em 27 DE na enfermaria de pediatria, separados por um diagnóstico de promoção da saúde, 12 diagnósticos de risco e 14 diagnósticos com foco no problema(15).

O DE é considerado um julgamento clínico sobre uma resposta humana a condições de saúde/processos de vida, ou à vulnerabilidade para essa resposta por um indivíduo, família, grupo ou comunidade. Constitui-se a base para a escolha de intervenções de enfermagem para alcançar resultados pelos quais respondem os enfermeiros ${ }^{(11)}$.

$O$ DE é a segunda fase do PE, e diagnosticar é um processo que exige conhecimento, habilidade de pensamento lógico, raciocínio, julgamento, interpretação, análise, previsão do significado e interação enfermeiro-paciente, sendo que a qualidade desta interação afeta diretamente a informação obtida. Sabe-se que os diagnósticos formulados só têm significação no contexto do PE se forem um elo entre o levantamento de dados e a determinação das intervenções necessárias para o alcance dos resultados esperados, não deixando que as ações prescritas se limitem apenas a cuidados de rotina, mas sim, cuidados consistentes e pertinentes com os diagnósticos de enfermagem elencados ${ }^{(16,11,17)}$.

Quanto ao diagnóstico de Promoção da Saúde, identificou-se apenas $O$ DE Proteção Ineficaz (diminuição na capacidade de proteger-se de ameaças internas ou externas, como doenças ou lesões) em 39 (39\%) dos pacientes internados. Destes, prevaleceram os pacientes da clínica de Onco-hematologia, com 21 $(21 \%)$ casos. Os casos classificados no estudo sob esse diagnóstico apresentavam as seguintes características definidoras: deficiência na imunidade, alteração na coagulação, prejuízo neurossensorial. E seus fatores relacionados: câncer, perfil sanguíneo anormal, distúrbios imunológicos ${ }^{(11)}$.

O DE de Promoção da Saúde é considerado o julgamento clínico sobre motivação e desejo de aumentar o bem-estar e concretizar o potencial de saúde humana. Essas respostas são expressas por uma disposição para melhorar comportamentos específicos de saúde e podem ser utilizadas em qualquer condição de saúde. Respostas de promoção da saúde podem ocorrer em individuo, família, grupo ou comunidade(11).

Encontrou-se 12 DE prevalentes de Risco, sendo que no DE Risco de Quedas houve frequência de $100 \%$, uma vez que a instituição considera que todos os pacientes internados em uma enfermaria de pediatria se encontram sob este risco. O Diagnóstico de Risco é compreendido como julgamento clínico sobre a vulnerabilidade de individuo, família, grupo ou comunidade para desenvolvimento de uma resposta humana indesejada a condições de saúde/processos de vida(1l) $^{(11}$

Identificou-se 14 DE prevalentes na categoria Foco no Problema, este considerado o julgamento clínico sobre uma resposta humana indesejada a condições de saúde/processos de vida, existente em individuo, familia, grupo ou comunidade(11).

Importante lembrar que diagnósticos com foco no problema não devem ser entendidos como mais importantes do que os de risco. Muitas vezes, um diagnóstico de risco pode ser o de maior prioridade para um paciente. Após identificação dos diagnósticos, 
deve-se priorizar os selecionados para determinar os cuidados prioritários. O diagnóstico prioritário é aquele com necessidade urgente, com alto nível de coerência com as características definidoras, fatores relacionados ou de risco e que necessita ser identificado para que o cuidado possa ser direcionado à solução desses problemas ou redução da gravidade ou risco de ocorrência ${ }^{(11)}$

É fundamental o envolvimento de toda a equipe de enfermagem na aplicação do PE a fim de identificar as necessidades biopsicoespirituais das crianças hospitalizadas, embasando o cuidado à criança e seus familiares de forma humanizada, sistematizada e integral. Nesse sentido, encontra-se a necessidade de estabelecer uma linguagem comum, padronizada, que seja utilizada universalmente pelos profissionais e adaptada às mais variadas culturas e contexto. Esta padronização busca qualificar o cuidado prestado, ofertar atendimento planejado e individualizado, manter a organização do setor, otimizar o tempo ao identificar as prioridades para a tomada de decisões, além de melhorar a comunicação entre os profissionais ${ }^{(6,18,19)}$.

Para a implementação da SAE de maneira eficiente e eficaz, é primordial o enfoque do eixo Pessoas, para que os profissionais enfermeiros tenham competências e habilidades desenvolvidas com base em conhecimentos científicos, éticos, técnicos e atitudinais, além de responsabilidade com o cuidado do paciente. Quanto às instituições de saúde, estas precisam realizar uma gestão dos serviços em que haja um dimensionamento adequado de pessoal, promoção de capacitações acerca da temática, além de estratégias facilitadoras capazes de otimizar a adesão dos profissionais e gestores ${ }^{(19)}$.

A implementação da SAE em sua totalidade consolida e respalda a profissão, proporciona, autonomia profissional, melhor qualidade no cuidado prestado ao paciente, diminuição no tempo de internação e, consequentemente, economia de recursos. A SAE possibilita ao enfermeiro a organização, planejamento e avaliação do cuidado prestado. Constitui-se, ainda, em estratégia fundamental para alcançar a qualidade da assistência, melhorar a comunicação entre a equipe, priorizar as necessidades de cada paciente, desenvolver ações baseadas em conhecimento técnico científico, evidenciando a segurança do paciente $e^{(19,4,20)}$.

\section{Limitação do estudo}

O estudo apresenta limitação metodológica e espacial por utilizar de dados secundários de uma única instituição hospitalar, que carecem de fidedignidade em relação aos registros feitos em portuários e fichas.

\section{Contribuições do estudo para a prática}

Conhecer o perfil sociodemográfico e clínico dos pacientes internados contribui para o estabelecimento de diagnóstico situacional da unidade como ferramenta de gestão da implantação da SAE. Os diagnósticos de enfermagem prevalentes na enfermaria de pediatria possibilitam a criação de uma proposta para prescrição de enfermagem, com os principais diagnósticos e intervenções de enfermagem para os pacientes internados na enfermaria de pediatria. Recomenda-se, portanto, a continuidade do processo de implantação do PE.

\section{CONCLUSÃO}

A SAE fundamentada em uma teoria de enfermagem e um sistema de linguagem padronizada norteia as etapas do PE, direcionando as ações de cuidado, além de contribuir para o fortalecimento da identidade profissional.

Com a realização do diagnóstico situacional da enfermaria de pediatria, por meio da caracterização do perfil dos pacientes, torna possível dar continuidade à implementação da SAE de forma integral na enfermaria. contribuindo para a melhora contínua da qualidade e segurança dos cuidados de enfermagem, permitindo uma assistência individualizada.

\section{Contribuição dos autores}

Concepção e/ou desenho: Reiniack S, Gonçalves JPF. Análise e interpretação dos dados: Reiniack S, Gonçalves JPF, Silva AS, Tonini T. Redação do artigo: Reiniack S, Gonçalves JPF. Revisão crítica: Silva AS Revisão final: Tonini T.

\section{FINANCIAMENTO}

Convênio CAPES (Coordenação de Aperfeiçoamento de Pessoal de Nivel Superior)/ COFEN (Conselho Federal de Enfermagem) 


\section{REFERÊNCIAS}

1. Souza ATF. Proposta de instrumento para avaliação da implantação da sistematização da assistência de enfermagem - SAE: construção e validação. [Dissertação]. Rio de Janeiro: Universidade Federal do Estado do Rio de Janeiro; 2016.

2. Tannure MC, Pinheiro AM. SAE: Sistematização da Assistência de Enfermagem: Guia Prático. 2 ed. Rio de Janeiro: Guanabara Koogan: 2011.

3. Benedet SA, Gelbcke FL, Amante LN, Padilha MIS, Pires DP. Nursing process: systematization of the nursing care instrument in the perception of nurses. $\mathrm{J}$ res fundam care online [Internet]. 2016 [cited 2018 Oct 5]:8(3). Available from: http://www.seer.unirio.br/index.php/cuidadofundamental/article/view/4237

4. Brasil. Conselho Federal de Enfermagem. Resolução n.o 358, de 15 de outubro de 2009. Dispõe sobre a sistematização da assistência de enfermagem e a implementação do processo de enfermagem em ambientes, públicos ou privados, em que ocorre o cuidado profissional de Enfermagem, e dá outras providências. Diário Oficial [da República Federativa do Brasil]. Brasilia, Oct 2009 [cited 2017 Apr 10]. Available from: http://www.cofen.gov.br/resoluo-cofen3582009_4384.html

5. Luvisaro BMO, Lima GS, Freire EMR, Martinez MR. Diagnóstico situacional em unidade de terapia intensiva: relato de experiência. Revista de Administração Hospitalar e Inovação em Saúde - RAHIS [Internet]. 2014 [cited 2018 Sept 3]:11(2). Available from: https://revistas.face.ufmg.br/index.php/rahis/article/view/2012

6. Andrade JS, Mattos MCT, Vieira MJ, organizadoras. Experiências em Sistematização da Assistência de Enfermagem. Aracaju: editora; 2016 [cited 2018 Oct 10]. Available from: http://se.corens.portalcofen.gov.br/wp-content/uploads/2017/02/parte-1.pdf

7. Silva RS, Almeida ARLP, Oliveira FA, Oliveira AS, Sampaio MRFB Paixão GPN. Sistematização da Assistência de Enfermagem na perspectiva da equipe. Enferm Foco [Internet]. 2016 [cited 2019 Jun 24]:7(2):32-36. Available from: http://revista.cofen.gov.br/index. php/enfermagem/article/view/803/328

8. Oliveira ALG, Silvino ZR. Prevalência de diagnósticos de enfermagem NANDA-I em um hospital pediátrico. Nursing (São Paulo) [Internet]. 2017 Ago [cited 2018 Sept 22]:20(231):1792-1796. Available from: http://pesquisa.bvsalud.org/enfermagem/resource/pt/ bde-31482

9. Chaves LD, Solai C. Sistematização da assistência de enfermagem: considerações teóricas e aplicabilidade. 2 ed. São Paulo: Martinari; 2015.

10. Ferreira AM, Rocha EN, Lopes CT, Bachion MM, Lopes JL, Barros ALBL. Diagnósticos de enfermería en terapia intensiva: mapeo cruzado y taxonomía de la NANDA-I. Rev Bras Enferm. [Internet]. 2016 Mar/Apr [cited 2018 Sept 5]:69(2):307-15. Available from: http:// www.scielo.br/pdf/reben/v69n2/0034-7167-reben-69-02-0307.pdf

11. Nanda International. Diagnósticos de Enfermagem da NANDA: definições e classificação 2015-2017. Tradução: Regina Machado
Garcez. 10ª ed. Porto Alegre: Artmed; 2015

12. INCA. Instituto Nacional de Câncer. Incidência, mortalidade e morbidade hospitalar por câncer em crianças, adolescentes e adultos jovens no brasil: informações dos registros de câncer e do sistema de mortalidade [Internet]. Brasil; 2017 [cited 2019 Feb 11] Available from: http://wwwl.inca.gov.br/wcm/incidencia/2017/pdf/ incidencia.pdf

13. Dini AP. Guirardello EB. Sistema de classificação de pacientes pediátricos: aperfeiçoamento de um instrumento. Rev esc enferm USP [Internet]. 2014 [cited 2018 Aug 12]:48(5):787-93. Available from: http://www.scielo.br/pdf/reeusp/v48n5/pt_0080-6234-reeusp-48-05-787.pdf

14. Brasil. Conselho Federal de Enfermagem. Resolução n.o 543, de 18 de abril de 2017. Atualiza e estabelece parâmetros para o Dimensionamento do Quadro de Profissionais de Enfermagem nos serviços/locais em que são realizadas atividades de enfermagem. Diário Oficial [da República Federativa do Brasil]. Brasilia, Apr 2017 [cited 2018 Oct 10]. Available from: http://www.cofen.gov.br/resolucao-cofen-5432017_51440.html

15. Reichert APS, Rodrigues PF, Albuquerque TM, Collet N, Minayo MCS. Bond between nurses and mothers of children younger than two years: perception of nurses. Ciênc saúde coletiva [Internet] 2016 [cited 2018 Aug 5];21(8):2375-2382. Available from: http://www. scielo.br/pdf/csc/v2ln8/1413-8123-csc-21-08-2375.pdf

16. Cruz AF, Rodriguez SP. Fernández IDP, Rabi MH. Sistema de acciones para desarrollar la habilidad diagnosticar en el Proceso de Atención de Enfermeri. Humanidades Médicas [Internet]. 2015 [cited 3018 Aug 10];15(2):294-306. Available from: https://www.medigraphic.com/cgi-bin/new/resumen.cgi?IDARTICULO=60488

17. Paiano LAG, Matos FGOA, Richetti MAA, Casarolli ACG, Girardello DTF, Barbosa HB, et al. Padronização das ações de enfermagem prescritas para pacientes clínicos e cirúrgicos em um hospital universitário. R Enferm Cent O Min. [Internet]. 2014 [cited 2018 Nov 11]:4(3). Available from: http://www.seer.ufsj.edu.br/index.php/recom/article/view/557

18. Barros ALBL, Sanchez CG, Lopes JL, Dell'Acqua MCQ, Lopes MHBM, Silva RCG; Conselho Regional de Enfermagem de São Paulo. Processo de enfermagem: guia para a prática. São Paulo: COREN-SP: 2015 [cited 2018 Sept 20]. Available from: http://www.coren-sp.gov.br/sites/default/files/SAE-web.pdf

19. Kirchesch CL. Estratégias para implementar a sistematização da assistência de enfermagem nos serviços de saúde: revisão integrativa. R Interd. [Internet]. 2016 [cited 2019 Jan 11]:9(4):173-180. Available from: https://revistainterdisciplinar.uninovafapi.edu.br/index $\mathrm{php} /$ revinter/article/view/1100

20. Pereira GN, Abreu RNDC, Bonfim IM, Rodrigues AMU, Monteiro LB, Sobrinho JM. Relação entre sistematização da assistência de enfermagem e segurança do paciente. Enferm Foco [Internet]. 2017 [cited 2019 Jun 24]:8(2):21-25. Available from: http://revista.cofen. gov.br/index.php/enfermagem/article/view/985/389 